\title{
Tumor suppressive IncRNA MEG3 binds to EZH2 and enhances CXCL3 methylation in gallbladder cancer
}

\author{
De-Qiang LI*, Yi-Ren DING*, Jin-Hui CHE, Zhan SU, Wei-Zhong YANG, Lu XU, Yun-Jiu LI, Hai-Hong WANG*, Wu-Yuan ZHOU* \\ Department of Hepato-Pancreato-Biliary Surgery, Xuzhou Cancer Hospital, Xuzhou, Jiangsu, China \\ ${ }^{*}$ Correspondence: wanghaihong_dr@163.com \\ ${ }^{*}$ Contributed equally to this work.
}

Received May 21, 2018 / Accepted July 22, 2019

\begin{abstract}
Gallbladder cancer is a malignant tumor with a high mortality rate. Accumulating evidence supports that lncRNA MEG3 may halt the progression of gallbladder cancer, while the downstream mechanism is rarely studied. Thus, we aim to investigate the molecular basis of the tumor-suppressing role of lncRNA MEG3 in gallbladder cancer. The expression of lncRNA MEG3 and CXCL3 was measured in patient serum and cell lines of gallbladder cancer. The viability, apoptosis, migration, and invasion of gallbladder cancer cells were assessed following ectopic MEG3 expression, as detected by CCK-8, flow cytometry, and Transwell assays. The interaction among lncRNA MEG3, EZH2, and CXCL3 was explored through ChIP, RNA pull-down, and RIP assays. The effects of lncRNA MEG3 and CXCL3 on tumor growth were evaluated by a mouse xenograft model. lncRNA MEG3 was expressed at a low level in gallbladder cancer patient serum and cell lines, while CXCL3 was highly expressed. MEG3 overexpression repressed the malignant behaviors of gallbladder cancer cells and promoted their apoptosis. MEG3 was mainly localized in the nucleus. MEG3 bound to EZH2, and EZH2 catalyzed the H3K27 trimethylation of the CXCL3 promoter region. MEG3 downregulated CXCL3 by activating EZH2-mediated H3K27 trimethylation of CXCL3; MEG3 overexpression attenuated cancer cell malignant behaviors in vitro and suppressed tumor growth in vivo in gallbladder cancer by inhibiting CXCL3 expression. Altogether, our results indicate that lncRNA MEG3 impedes gallbladder cancer development via the EZH2-CXCL3 axis, offering potential biomarkers for gallbladder cancer management.
\end{abstract}

Key words: gallbladder cancer; IncRNA MEG3; enhancer of zeste homolog 2; CXCL3; histone methylation; H3K27me3

Gallbladder cancer is a tremendously aggressive carcinoma, which is developed from the biliary tract system $[1,2]$. Since early symptoms are difficult to notice, most gallbladder cancer cases are diagnosed at late stages [3]. Despite advances in surgical treatment and chemotherapy for gallbladder cancer, the overall prognosis of gallbladder cancer patients remains poor [4]. The last decades have witnessed the identification of many tumor-promoting oncogenes and tumor suppressors, but few independent biomarkers are routinely employed in clinical therapies $[5,6]$. Therefore, it is vital to identify novel targets that can serve as promising biomarkers for the diagnosis and treatment of gallbladder cancer and to further explore underlying molecular mechanisms.

Notably, long noncoding RNAs (lncRNAs) play critical roles in the physiological and pathological progression of a wide spectrum of cancers including glioblastoma [7], abdominal aortic aneurysm [8], lung cancer [9, 10], and also in gallbladder cancer $[6,11]$. Recently, accumulating evidence has illustrated that lncRNAs are involved in the regulation of many malignant tumor processes such as ferroptosis and apoptosis [12-15]. It is interesting to note that lncRNA maternally expressed gene 3 (MEG3) is poorly expressed in many tumor tissues and acts as a suppressor for various cancers $[16,17]$. In addition, it has been demonstrated that lncRNA MEG3 is under-expressed in gallbladder cancer cells and tissues, as well as associated with a poor prognosis of gallbladder cancer patients [18]. However, the specific effects of lncRNA MEG3 on gallbladder cancer and its downstream mechanism need further investigation.

Enhancer of zeste homolog 2 (EZH2), a conserved histone methyltransferase, could act as a trigger of lysine 27 of histone $\mathrm{H} 3$ (H3K27me3) trimethylation and then inhibit the translation of target genes, which is involved in multiple biological processes, such as cell proliferation, cell cycle regulation and tumorigenesis [19]. Besides, many lncRNAs interact with EZH2 and further regulate downstream genes [20, 21]. 
Jin et al. have reported that MEG3 could degrade EZH2 by promoting its ubiquitination [18]. Notably, CXCL3, a member of the CXC-type chemokine family, plays a significant role in numerous human malignancies [22, 23]. For example, CXCL3 was illustrated to be expressed at a high level in breast cancer, and closely related to breast cancer metastasis, so it may serve as a viable target for cancer management [24]. Therefore, the purpose of this study is to explore the effect of the lncRNA MEG3 on the growth and metastasis of gallbladder cancer with the potential involvement of EZH2 and CXCL3.

\section{Patients and methods}

Ethics statement. All involved participants signed written informed consents. The clinical trial research was conducted in accordance with the Declaration of Helsinki and approved by the Ethics Committee of the Xuzhou Cancer Hospital. The procedures of animal experiments were in compliance with the Guide for the Care and Use of Laboratory Animals and approved by the Ethics Committee of the Xuzhou Cancer Hospital.

Bioinformatics analysis. A gallbladder cancer-related microarray (GSE74048) along with the annotation file was retrieved from the GEO database. The microarray contained 3 normal samples (normal) and 3 gallbladder cancer samples (cancer), wherein differentially expressed genes (DEGs) were identified using the "limma" package in $\mathrm{R}$ language with $|\log \mathrm{FC}|>1$ and $\mathrm{p}<0.05$ as the screening threshold. Then, the "heatmap" package in R language was utilized to obtain the top ten DEGs for the construction of a heatmap. The expression of these candidate genes in cancer and normal samples was compared through the GEPIA tool.

Study subjects. From December 2014 to December 2016, 60 patients ( 15 males and 45 females, aged 36-87 years with a mean age of $77.97 \pm 7.49$ years, body mass index $=20.67-$ $25.41 \mathrm{~kg} / \mathrm{m}^{2}$ ) were enrolled in this study, who were pathologically diagnosed with gallbladder cancer in Xuzhou Cancer Hospital. According to the classification criteria described in a previous study [25], these gallbladder cancer patients included 23 cases of stage I, 18 cases of stage II, 13 cases of stage III, and 6 cases of stage IV. Meanwhile, 60 healthy individuals ( 17 males and 43 females, aged $36-87$ years with a mean age of $76.53 \pm 8.75$ years), who had come to the Xuzhou Cancer Hospital for physical examinations, were recruited as the healthy controls. Subsequently, $4 \mathrm{ml}$ of anticoagulated venous blood from gallbladder cancer patients and healthy controls was collected under fasting conditions. Patients were followed up by telephone interview for two years (ended on December 31, 2018), with their prognosis conditions (including the presence or absence of tumor recurrence and metastasis) recorded.

RNA quantitation. The Trizol method was employed to extract total RNA from serum, and the concentration and purity of the RNA were measured by a spectrophotometer. Oligo (dT) primers and M-MLV reverse transcriptase were used to reversely transcribe the RNA into cDNA. Subsequently, the cDNA was stained with SYBR GreenII (NH129155, Beijing Obit Instrument, Beijing, China) and then subjected to qRT-PCR detection. The expression of genes to be tested was quantified by the $2^{-\triangle \Delta C T}$ method, normalized to $\beta$-actin mRNA expression. Primers for the qRT-PCR assay were listed in Supplementary Table S1.

Western blot. RIPA lysis buffer (P22736, Aviva Systems Biology, San Diego, CA, USA) was adopted for protein extraction, and a BCA kit (MAB1835, R\&D Systems, Minneapolis, $\mathrm{MN}$, USA) was used for quantitative analysis of protein expression. Total proteins were extracted, separated by $12 \%$ SDS-PAGE, and then transferred to a PVDF membrane. Following this, the blot was incubated overnight with primary antibodies: rabbit anti-mouse CXCL3 (1:1000, ab220431), rabbit anti-mouse Ki67 (1:1000, ab16667), rabbit anti-mouse PCNA (1:1000, ab92552), rabbit anti-mouse EZH2 (1:1000, ab186006), and monoclonal anti- $\beta$-actin (1:1000, ab8227). Antibodies were all purchased from Abcam (Cambridge, UK). On the next day, secondary antibody goat anti-rabbit $\operatorname{IgG}(1: 2000, \mathrm{ab6721})$ was added to the membrane and incubated at room temperature for $2 \mathrm{~h}$. Subsequently, after three washes with Tris-buffered saline (TBS), the bands were visualized by enhanced chemiluminescence (ECL) solution. Finally, the gray values of bands were quantified by ImageJ software.

Cell culture and treatment. Gallbladder cancer cell lines GBC-SD (MZ-0066, Ningbo Mingzhou Biotechnology Co., Ltd., Ningbo, China), NOZ (iCell-h165, iCell Bioscience, Shanghai, China), SGC-996 (MZ-1916, Ningbo Mingzhou Biotechnology Co., Ltd., Ningbo, China), and normal gallbladder epithelial cells HGBEC (HTX-3131, Otwo Biotech Inc. Shenzhen, China) were cultured for 48 $\mathrm{h}$ at $37^{\circ} \mathrm{C}$ with $5 \% \mathrm{CO}_{2}$. By using Lipofectamine2000 transfection reagent (Thermo Fisher Scientific, Waltham, MA), GBC-SD cells were transduced with the eukaryotic lncRNA MEG3 expression vector (pCDNA3.1-MEG3), pLKO vector carrying shRNA targeting CXCL3 (sh-CXCL3), CXCL3 overexpression plasmid (oe-CXCL3), EZH2 overexpression plasmid (oe-EZH2), and EZH2 inhibitor EPZ-6438, alone or in combination, as well as corresponding controls oe-negative control (NC), sh-NC, and DMSO.

Cell viability assay. Cells in the logarithmic growth phase were seeded into 96 -well plates at a density of $5 \times 10^{3}$ cells/well and then incubated at $37^{\circ} \mathrm{C}$ and $5 \% \mathrm{CO}_{2}$. The culture medium was renewed on the next day. The cells were incubated with a culture medium containing $10 \mu \mathrm{l}$ of CCK-8 solution respectively at $24 \mathrm{~h}, 48 \mathrm{~h}, 72 \mathrm{~h}$, and $96 \mathrm{~h}$. After incubation for $3 \mathrm{~h}$, the optical density (OD) value at $450 \mathrm{~nm}$ was measured with a microplate reader (Safire2, Tecan, Männedorf, Switzerland), and then cell survival rate and half inhibitory concentration (IC50) were calculated.

Flow cytometric analysis. The Annexin V/propidium iodide (PI) apoptosis detection kit (BD Biosciences, Franklin Lakes, NJ, USA) was utilized to detect the apoptosis rate of 
cells in each group. After PBS washing, the cells were resuspended in binding buffer and incubated with $5 \mu$ l FITCAnnexin V and $5 \mu \mathrm{PI}$ at room temperature for $15 \mathrm{~min}$, followed by flow cytometry analysis using the FACScan system (Becton Dickinson, San Diego, CA, USA).

Transwell assays. Matrigel (Sigma-Aldrich, St. Louis, MO, USA) was used for Transwell invasion and migration assays of the aforementioned stably transfected GBC-SD cells. When conducting the invasion assay, the upper chamber of the Transwell plate was coated with Matrigel and filled with $100 \mu \mathrm{l}$ serum-free RPMI 1640. After incubation, the migrated cells were fixed with $4 \%$ paraformaldehyde for $15 \mathrm{~min}$ and then stained with $0.1 \%$ crystal violet. Cells of each chamber were observed under a microscope and six random fields were selected for cell counting. Matrigel was not applied to the upper chamber of the Transwell plate when performing the migration assay, and the rest steps were the same as the invasion experiment. The experiments were independently repeated three times.

FISH assay. FISH analysis was adopted to evaluate the localization of IncRNA MEG3 expression in gallbladder cancer cells. The cells growing on slides were placed on the bottom of 2-well plates at a density of $5 \times 10^{3}$ cells/well and incubated for $24 \mathrm{~h}$. Upon reaching a confluence of $80 \%$, cells were blocked by $0.5 \%$ TritonX-100-containing prehybridization solution PBS at $37^{\circ} \mathrm{C}$ after fixation in $4 \%$ paraformaldehyde. Then lncRNA MEG3 probe was added into the mixture and incubated at $42^{\circ} \mathrm{C}$ without light exposure overnight. The hybridization region was stained by DAPI. An anti-fluorescence quencher was used to fix the climbing slide to the glass slide. The experimental specimens were observed under a laser confocal microscope (MetaSystems, Altlussheim, Germany).

RIP assay. RIP assay was conducted according to the instructions of Magna RIP ${ }^{\text {tw }}$ RNA-Binding Protein Immunoprecipitation Kit (Millipore, Billerica, MA, USA). When cells reached a confluence of $90 \%$, they were lysed in RIP lysis buffer. Besides, the cell extraction was incubated with RNAs magnetic beads, which were conjugated to anti-EZH2 antibody (\#5246, 1:300, Cell Signaling Technology, Danvers, MA, USA) or matched negative control antibody anti-IgG. In addition, RT-qPCR was employed to assess the MEG3 enrichment.

RNA pull-down assay. The pcDNA3.1-MEG3 plasmid was used as a template for in vitro transcription and biotin labeling. Biotinylated wild-type lncRNA MEG3 (pcDNA3.1MEG3), biotinylated mutant lncRNA MEG3, or biotinylated negative control (pcDNA3.1) was respectively transfected into the GBC-SD cells. After incubation, the cells were lysed and then mixed with streptavidin beads (Shanghai Ruishi Biological Technology, Shanghai, China). Further, EZH2 protein was eluted from beads and measured by western blot analysis.

Chromatin immunoprecipitation (ChIP) assay. Chromatin immunoprecipitation experiment was performed using EZ-Magna ChIP A kit (17-408, Upstate, Lake Placid, NY, USA). Gallbladder cancer cells, in the logarithmic growth phase $\left(1 \times 10^{7}\right.$ cells/dish), were incubated with $20 \mathrm{ml}$ of the whole medium (containing freshly prepared $37 \%$ formaldehyde) at room temperature for $10 \mathrm{~min}$. Then, the mixture was added with $10 \times$ glycine and incubated at room temperature for $5 \mathrm{~min}$ to terminate cross-linking. Subsequently, protease inhibitor Cocktail II was added to the mixture, and cells were harvested and sonicated to shear DNA. The supernatant was obtained and incubated with protein-G-coated magnetic beads, followed by incubation with IgG. RT-qPCR was employed to measure the expression of CXCL3 with characteristic primers of the CXCL3 gene promoter region, and the primers involved were listed in Supplementary Table S2. For experimental groups, anti-EZH2 (ab191080, 1:200) and antiH3K27me3 (ab6002, 1:200) antibodies were used, and IgG was used as NC.

Subcutaneous tumor growth assay. Eighteen 4- to 6-week-old Balb/c nude mice, weighing 18 to $22 \mathrm{~g}$, were obtained from Hunan SLAC Jingda Laboratory Animal (Changsha, Hunan, China) for tumorigenesis experiments. Nude mice were kept in a constant temperature $\left(25-27^{\circ} \mathrm{C}\right)$ and constant humidity (45-50\%) environment. They were randomly classified into 3 groups (6 nude mice in each group): pCDNA3.1+oe-NC group, pCDNA3.1-MEG3+oeCXCL3 group, and pCDNA3.1-MEG3+oe-NC group. Stably transfected GBC-SD cells in the logarithmic growth phase were trypsinized and inoculated into the dorsal surface of the right hind limb of mice. When the tumor grew to 1.0 $\mathrm{cm}$ in diameter, it was removed under sterile conditions. Then necrotic tissue and fibrous tissue were removed, and the tumor was cut into tumor blocks of about $1 \mathrm{~mm}^{3}$. The cell suspension was made by the above-mentioned tumor blocks and injected into the gallbladder of nude mice. The gallbladder was exposed and wiped to destroy its serous membrane surface. After inoculation, the growth condition of nude mice and tumors were observed every day and measured every 5 days. After 30 days of inoculation, the nude mice were euthanized. The tumors were extracted, with volume and weight measured. The lymph node metastasis and pathological section of liver tissue were observed.

Hematoxylin and eosin (HE) staining. The pathological changes of tumor lesions were observed by HE staining. The tumor tissue was taken out and fixed in $10 \%$ formaldehyde, dehydrated, permeabilized, and embedded with paraffin. The slices were dehydrated by gradient alcohol, permeabilized with xylene, and then stained with hematoxylin. Subsequently, eosin solution (PT001, Shanghai Bogu Biotechnology, Shanghai, China) was used to stain slices, which were then mounted in a neutral gum. Then, the histopathological changes of tumor xenografts were observed under an optical microscope.

Immunohistochemistry assay. After paraffin embedding, the tumor tissue was sectioned into slices with $4 \mu \mathrm{m}$ thickness. After dewaxing with xylene and hydration with 
alcohol, the sections were treated with high temperature for antigen retrieval. Subsequently, the sections were blocked with non-immune mouse serum and then incubated with CXCL3 rabbit monoclonal antibody at $4{ }^{\circ} \mathrm{C}$ overnight. After PBS washes, rabbit anti-mouse IgG was employed to incubate sections at room temperature for $15 \mathrm{~min}$. Further, the sections were colored with DAB. The positive expression of CXCL3 was observed under an optical microscope and 5 fields were randomly selected to count the cells.

Statistical analysis. The statistical analyses were conducted by SPSS 21.0 (IBM SPSS Statistics, Chicago, IL, USA). The measurement data were expressed as mean \pm standard deviation. An unpaired t-test was used to analyze the unpaired data in the two groups that were subject to the normal distribution and equal variance. One-way analysis of variance (ANOVA) with Tukey's post-hoc test was adopted to compare data between multiple groups. The data at different time points were compared by repeated-measures ANOVA followed by Bonferroni post-hoc test. The survival rate of patients was analyzed by the Kaplan-Meier method, with the log-rank test adopted for univariate analysis. Statistical significance was defined at a level of $5 \%(\mathrm{p}<0.05)$.

\section{Results}

lncRNA MEG3 is poorly expressed in patent serum and cells of gallbladder cancer. In order to study the relationship between lncRNA MEG3 and gallbladder cancer, we examined the expression level of lncRNA MEG3 in the serum of gallbladder cancer patients. As shown in Figure 1A, after measurement of RT-qPCR, lncRNA MEG3 was expressed at low levels in patients with gallbladder cancer compared with that in healthy controls. Subsequently, we found that in comparison with normal gallbladder epithelial cells (HGBEC), lncRNA MEG3 was highly expressed in gallbladder cancer cell lines including GBC-SD, SGC-996,
A

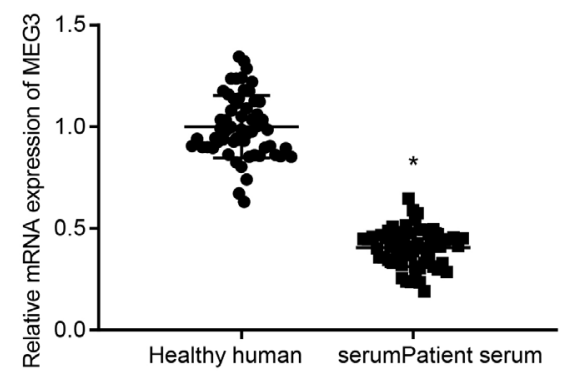

B

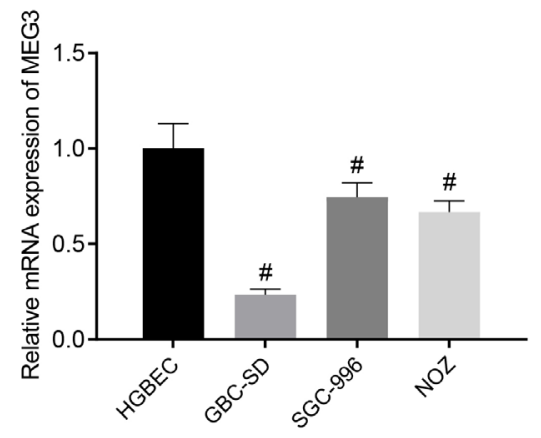

c

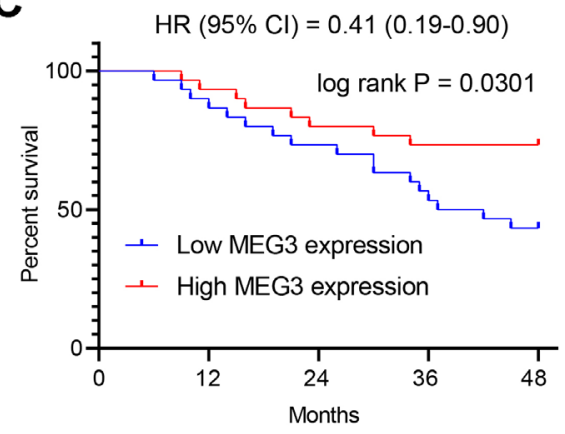

D

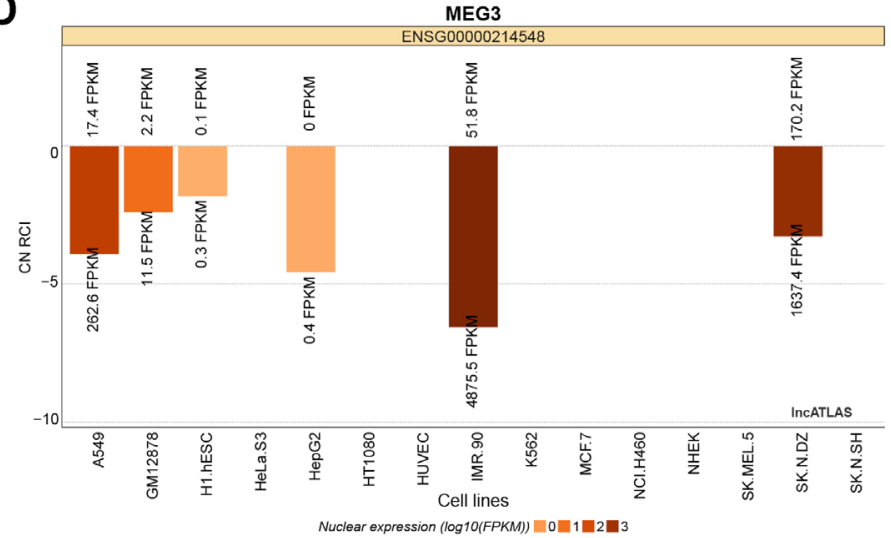

E
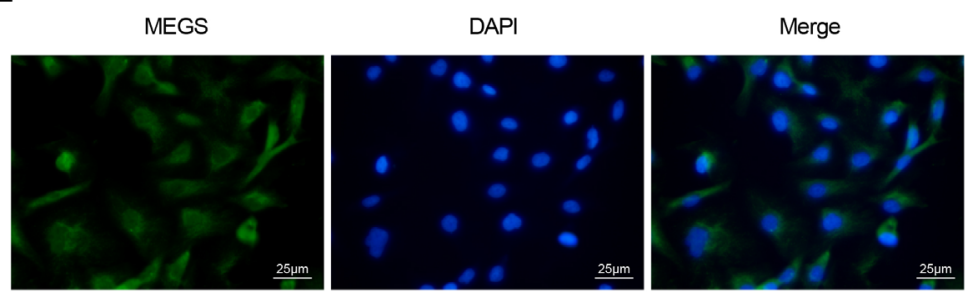

Figure 1. IncRNA MEG3 was under-expressed in patient serum and cell lines of gallbladder cancer. A) The expression of IncRNA MEG3 in serum of gallbladder cancer patients and healthy controls, as measured by RT-qPCR. Healthy controls: $n=60$; gallbladder cancer patients: $n=60$. ${ }^{*} p<0.05$ compared with healthy controls. $\left.B\right)$ The expression of lncRNA MEG3 in HGBEC cells and gallbladder cancer cell lines GBC-SD, SGC-996, and NOZ, as measured by RT-qPCR. C) Kaplan-Meier survival analysis to detect the correlation between the survival in patients and MEG3 expression level, with patients divided into two groups according to higher/lower expression of MEG3. D) Bioinformatics analysis of the subcellular localization of MEG3. E) FISH assay to detect the subcellular localization of MEG in gallbladder cancer cells. \#p $<0.05$ relative to HGBEC; \#\# $<<0.01$ relative to HGBEC cells. Measurement data were shown as mean \pm standard deviation, with unpaired t-test, one-way ANOVA with Tukey's post-hoc test. Cell experiments were repeated in triplicate. 
and NOZ, among which GBC-SD showed the lowest level (Figure 1B). Thus, the GBC-SD cell line was selected for subsequent experiments.

Moreover, Kaplan-Meier survival analysis showed that patients with a relatively lower MEG3 expression level presented with a shorter survival time relative to those with higher expression MEG3 level (Figure 1C). Further, the subcellular localization of MEG3 was predicted through the lncATLAS database (http://lncatlas.crg.eu/), and the results displayed that MEG3 was mainly located in the nucleus (Figure 1D). Results of the FISH assay then confirmed that MEG3 was mainly located in the nucleus of gallbladder cancer cells (Figure 1E).

IncRNA MEG3 overexpression inhibits the viability, migration, and invasion of gallbladder cancer cells and enhances apoptosis. Next, we proceeded to explore the effect of IncRNA MEG3 on gallbladder cancer cell activities. As shown in Figure 2A, the expression of lncRNA MEG3 was significantly elevated in response to the pCDNA3.1MEG3 treatment as compared with pCDNA3.1 treatment, suggesting qualified transduction efficiency. Afterward, we found that lncRNA MEG3 overexpression inhibited the viability of gallbladder cancer cells, as assessed by the CCK- 8 assay (Figure 2B). Besides, cell apoptosis was augmented in the presence of lncRNA MEG3 overexpression, as shown by flow cytometric data (Figure 2C). In addition, lncRNA MEG3 overexpression suppressed the migration and invasion ability of gallbladder cancer cells, as evaluated by Transwell assays (Figure 2D). Western blot assay was then performed to determine the expression of proliferation-related proteins (Ki67, PCNA), apoptosis-related proteins (Bcl2, Bax), invasion and migration-related proteins (E-cadherin, MMP2, and MMP7). The results displayed that MEG3 overexpression led to the downregulated expression of pro-proliferative Ki67 and PCNA, anti-apoptotic Bcl2, and pro-invasive, pro-migratory E-cadherin as well as upregulated expression of pro-apoptotic Bax and anti-invasive, anti-migratory MMP2 and MMP7 (Figure 2E). In summary, overexpression of MEG3 can reduce cell proliferation, migration, and invasion, and promote cell apoptosis.

CXCL3 silencing inhibits gallbladder cancer cell malignant phenotypes and promotes cell apoptosis. Following the aforementioned results, we identified 1,742 DEGs related to gallbladder cancer through the differential analysis of the GSE74048 microarray dataset (Figure 3A). A heat map was then constructed to show the expression of the 10 most differential DEGs, among which CXCL3 was found to be highly expressed in gallbladder cancer samples (Figure 3B). Subsequent expression analysis utilizing the GEPIA website also revealed the upregulated expression of CXCL3 in gallbladder
A
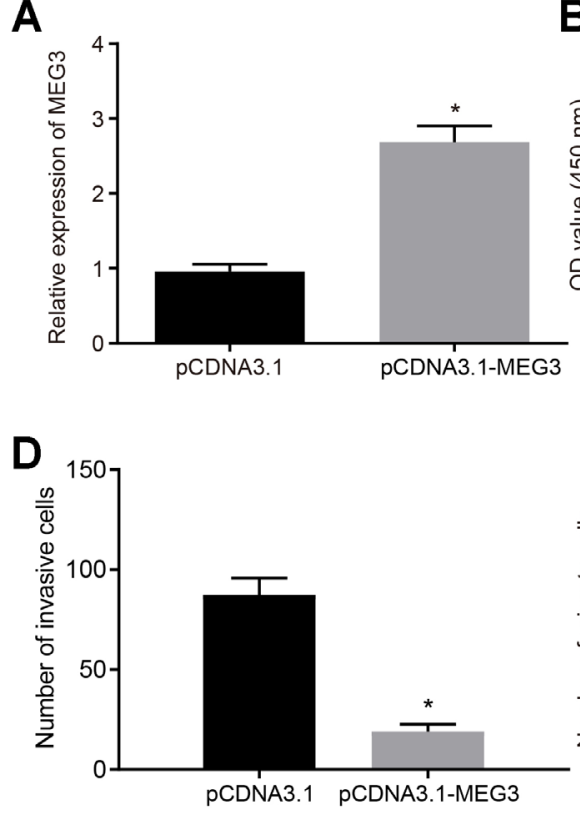
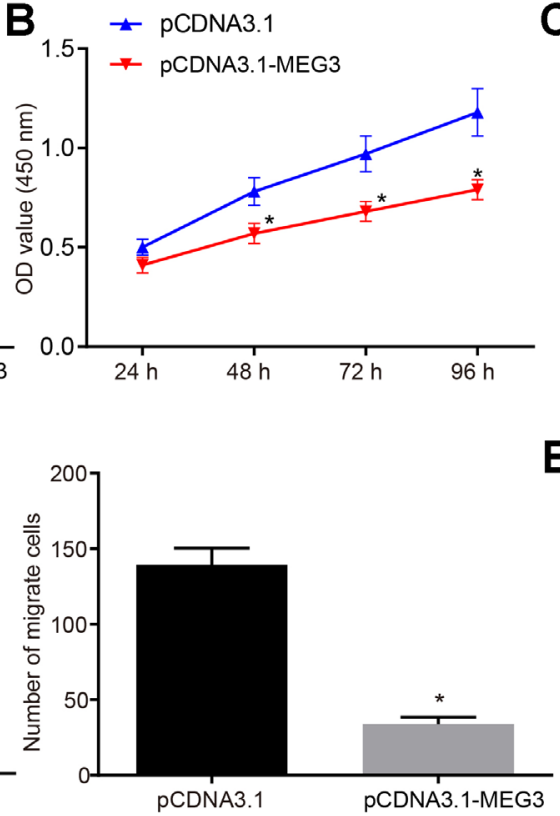

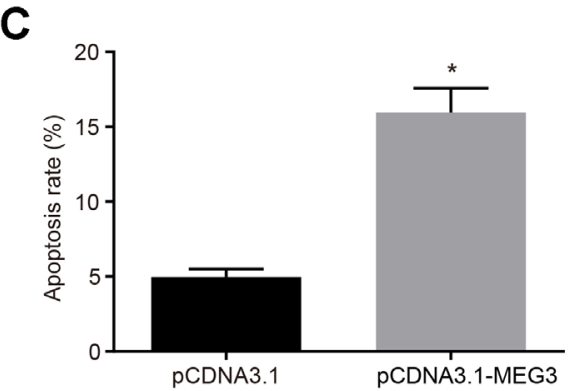

E

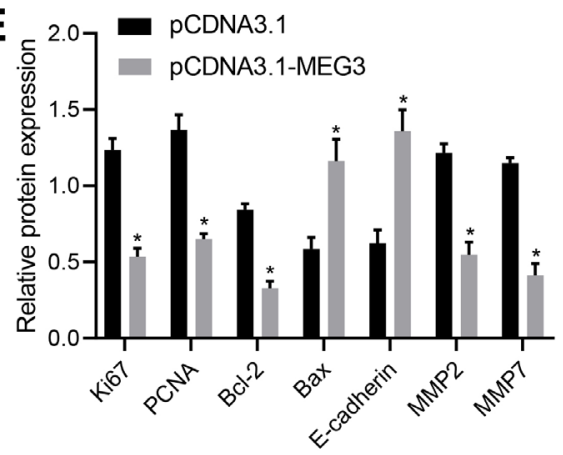

Figure 2. IncRNA MEG3 restricted the viability, migration, and invasion, and promoted apoptosis of gallbladder cancer cells. A) The expression of IncRNA MEG3 in the pCDNA3.1-MEG3 group and the pCDNA3.1 group. B) The viability of gallbladder cancer cells in response to lncRNA MEG3 overexpression, as measured by CCK-8 assay. C) The apoptosis of gallbladder cancer cells in the presence of IncRNA MEG3 overexpression, as assessed by flow cytometry. D) The migration and invasion ability of gallbladder cancer cells in the presence of IncRNA MEG3 overexpression, as assessed by Transwell assays. E) Western blot measurement of the expression of proteins related to proliferation, invasion, migration, and apoptosis in response to MEG3 overexpression. ${ }^{\star} \mathbf{p}<0.05$ relative to the $\mathrm{pCDNA3.1}$ group. Measurement data were expressed as mean \pm standard deviation, with unpaired $\mathrm{t}$-test and repeated measures ANOVA followed by Bonferroni post-hoc test. Cell experiments were repeated three times. 

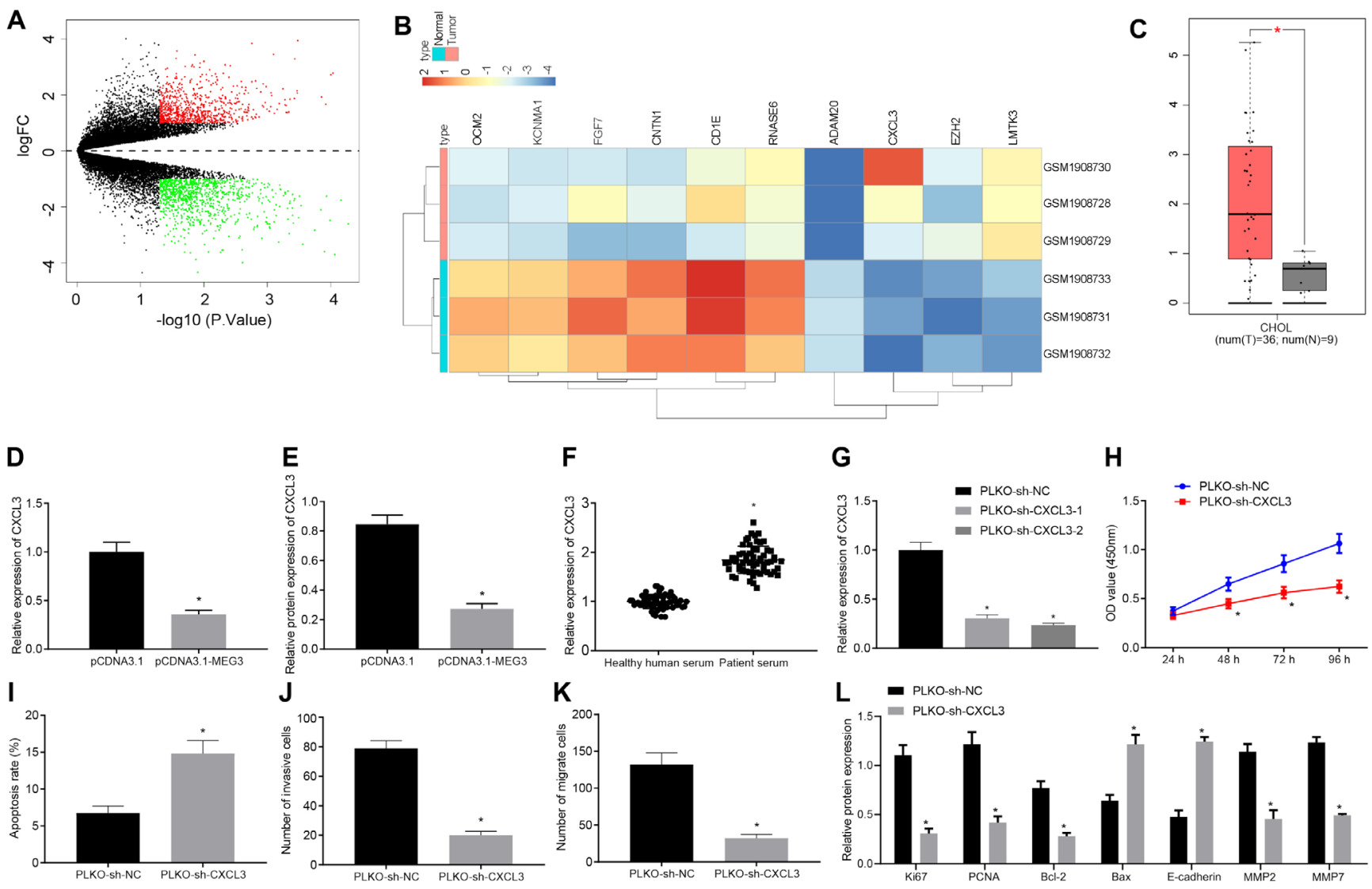

Figure 3. CXCL3 silencing inhibited malignant behaviors of gallbladder cancer cells. A) The volcano plot of differentially expressed genes (DEGs) in the GSE74048 gallbladder cancer-related microarray (red dots indicate highly expressed DEGs, green dots indicate poorly expressed DEGs, the Xaxis indicates $\mathrm{p}$ value, and the Y-axis indicates $\operatorname{logFC}$ ). B) The heat map of the expression of the top 10 most significant DEGs. C) The box diagram of CXCL3 expression in gallbladder cancer samples and normal samples, analyzed by the GEPIA tool (* $|\log 2 \mathrm{FC}|>1, \mathrm{p}<0.01)$. D) RT-qPCR measurement of the expression of CXCL3 in response to MEG3 overexpression. E) Western blot measurement of the expression of CXCL3 in response to MEG3 overexpression. F) RT-qPCR measurement of CXCL3 expression in the serum of patients with gallbladder cancer. G) RT-qPCR measurement of CXCL3 expression in gallbladder cancer cells treated with sh-CXCL3-1 or sh-CXCL3-2 ( ${ }^{*} \mathbf{p}<0.05$ versus the PLKO-sh-NC group). H) The viability of gallbladder cancer cells in response to CXCL3 silencing, detected by CCK-8 assay $\left({ }^{*} \mathbf{p}<0.05\right.$ versus the PLKO-sh-NC group). I) The apoptosis of gallbladder cancer cells in response to CXCL3 silencing, detected by flow cytometry $\left({ }^{*} \mathrm{p}<0.05\right.$ versus the PLKO-sh-NC group). J) The invasion of gallbladder cancer cells in response to CXCL3 silencing, detected by Transwell assay ( ${ }^{*} \mathrm{p}<0.05$ versus the PLKO-sh-NC group). K) The migration of gallbladder cancer cells in response to CXCL3 silencing, detected by Transwell assay $\left({ }^{*} \mathrm{p}<0.05\right.$ versus the PLKO-sh-NC group). L) Western blot measurement of proteins related to proliferation, migration, and invasion in the presence of CXCL3 silencing $\left({ }^{*} \mathrm{p}<0.05\right.$ versus the PLKO-sh-NC group). Measurement data were expressed as mean \pm standard deviation, with unpaired t-test and repeated-measures ANOVA followed by Bonferroni post-hoc test. Cell experiments were repeated three times.

cancer samples relative to normal samples (Figure 3C). Furthermore, we demonstrated through RT-qPCR and western blot measurement that MEG3 overexpression led to downregulated mRNA and protein levels of CXCL3 (Figures 3D, 3E).

Subsequently, we moved to study the effect of CXCL3 on gallbladder cancer cell activities. By RT-qPCR, we illustrated that CXCL3 was overexpressed in the serum of gallbladder cancer patients compared with that of healthy controls (Figure 3F). Further, in order to investigate the relationship between lncRNA MEG3 and CXCL3, we knocked CXCL3 down. CXCL3 expression was validated to be downregulated in response to sh-CXCL3 (sh-CXCL3-1, sh-CXCL3-2) treat- ment; sh-CXCL3-2 exhibited a better silencing efficiency relative to sh-CXCL3-1 and was thus selected for subsequent experiments (Figure 3G). The viability of gallbladder cancer cells, detected by CCK-8 assay, was inhibited in response to CXCL3 silencing (Figure $3 \mathrm{H}$ ). The apoptosis of the cells was shown by flow cytometry to be augmented in response to CXCL3 knockdown (Figure 3I). The migration and invasion of the cells, as reflected by Transwell detection, were attenuated in the presence of CXCL3 silencing (Figures 3J, $3 \mathrm{~K})$. Moreover, CXCL3 knockdown resulted in downregulated expression of pro-proliferative Ki67 and PCNA, antiapoptotic $\mathrm{Bcl} 2$, and pro-invasive, pro-migratory E-cadherin as well as upregulated expression of pro-apoptotic Bax and 
anti-invasive, anti-migratory MMP2 and MMP7 (Figure 3L). Collectively, our results suggested that CXCL3 inhibition could restrict malignant behaviors and trigger cell apoptosis in gallbladder cancer cells.

lncRNA MEG3 recruits $\mathrm{EZH} 2$ to the promoter of CXCL3 and further inhibits CXCL3 expression. The aforementioned experiments have illuminated that MEG3 can inhibit the CXCL3 expression. We then examined the speculation that MEG3 may regulate CXCL3 expression through, recruiting EZH2, a histone methyltransferase, and mediating EZH2-induced H3K27 methylation. We first observed the upregulated expression of EZH2 in gallbladder cancer utilizing the data retrieved from the GEPIA database (Supplementary Figure S1). Then, we determined the expression of EZH2 in the serum of gallbladder cancer patients, wherein EZH2 was found to be highly expressed relative to that in healthy controls (Figure 4A). Upregulated expression of EZH2 was also observed in gallbladder cancer cell lines (GBC-SD, SGC-996, and NOZ) as compared with normal gallbladder epithelial cells HGBEC (Figure 4B). Afterward, results of RNA pull-down and RIP assays illustrated that EZH2 could bind to lncRNA MEG3 (Figures 4C, 4D).
According to RT-qPCR results, either MEG3 overexpression or EZH2 overexpression led to decreased expression of CXCL3, and additional treatment by EZH2 inhibitor could reverse the decline in CXCL3 expression induced by MEG3 overexpression alone (Figure 4E). These results indicated that EZH2 may inhibit the transcriptional expression of CXCL3 in a way dependent on the H3K27me modification in the CXCL3 promoter region.

Subsequently, the ChIP assay was performed with EZH2 and $\mathrm{H} 3 \mathrm{~K} 27 \mathrm{me} 3$ antibodies, and the results demonstrated that silencing EZH2 and MEG3 can reduce the recruitment of EZH2 and $\mathrm{H} 3 \mathrm{~K} 27 \mathrm{me} 3$ to the CXCL3 promoter region (Figure 4F). Additionally, the ChIP assay was adopted to assess the level of CXCL3 binding to H3K27me3. Relative to that in the IgG group, the expression of the CXCL3 promoter region was enhanced in the $\mathrm{H} 3 \mathrm{~K} 27 \mathrm{me} 3$ antibody group; relative to the NC group, the expression of the CXCL3 promoter region was upregulated in the presence of MEG3 overexpression (Figure 4G), suggesting that the CXCL3 promoter region could directly bind to $\mathrm{H} 3 \mathrm{~K} 27 \mathrm{me} 3$ and that H3K27me3 modification did happen in the CXCL3 gene. Taken together, our data supported that MEG3 recruited
A

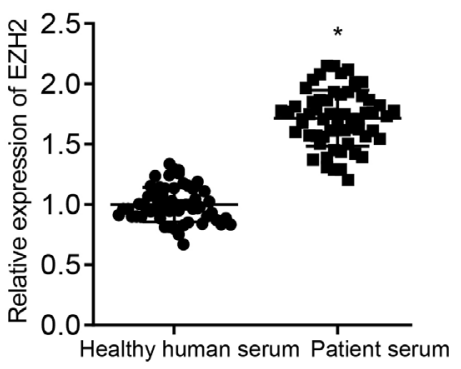

E

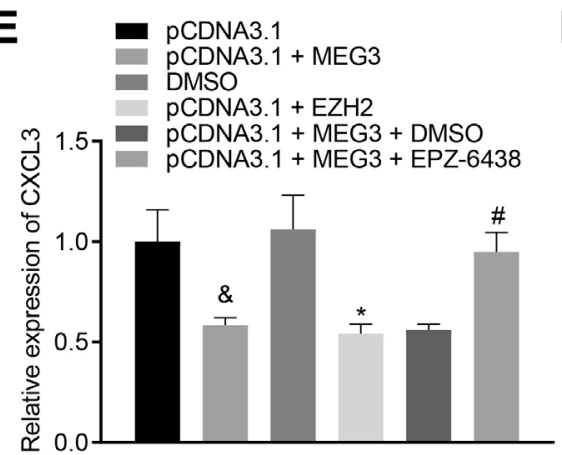

B

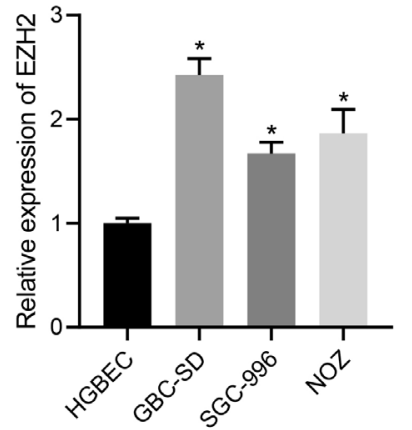

$\mathbf{F}$

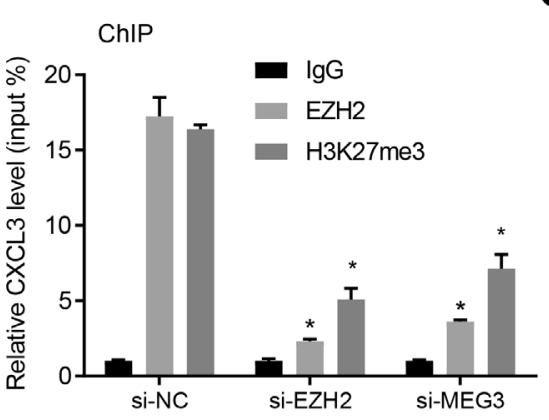

D

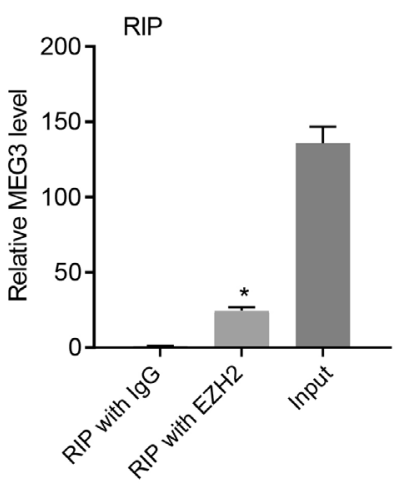

G

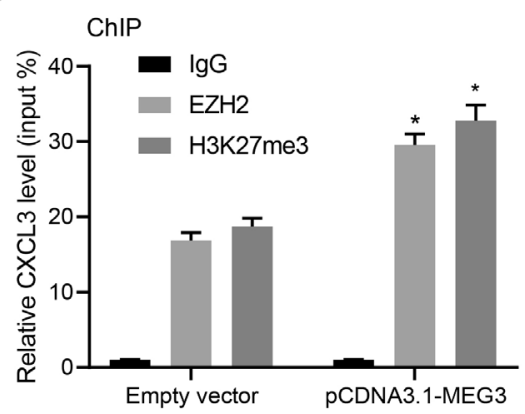

Figure 4. IncRNA MEG3 could bind to EZH2 and further repress CXCL3 expression. A) RT-qPCR measurement of the expression of EZH2 in the serum of patients with gallbladder cancer $\left({ }^{*} \mathrm{p}<\mathbf{0 . 0 5}\right.$ compared with healthy controls). B) RT-qPCR measurement of the expression of EZH2 in HGBEC cells and three gallbladder cancer cell lines GBC-SD, SGC-996, and NOZ ( ${ }^{\star} p<0.05$ compared with HGBEC cells). C) The interaction between lncRNA MEG3 and EZH2, as detected by RNA pull-down assay. D) RIP assay was adopted to evaluate the binding between EZH2 and MEG3. E) The transfection efficiency of EZH2 and its effect on CXCL3 transcription expression, as examined by $\mathrm{RT}-\mathrm{qPCR}$. ${ }^{*} \mathrm{p}<0.05$ relative to the DMSO group, \#p<0.05 relative to the pCDNA3.1-MEG3+DMSO group; \&p<0.05 relative to the pCDNA3.1 group). F) The recruitment of EZH2/H3K27me3 by the CXCL3 promoter region, as assessed by $\mathrm{ChIP}$ assay $\left({ }^{*} \mathrm{p}<0.05\right.$ relative to the si-NC group). G) The binding of the $\mathrm{CXCL} 3$ promoter region to EZH2/H3K27 $\mathrm{me} 3$, as evaluated by ChIP assay $\left({ }^{\star} \mathrm{p}<0.05\right.$ relative to the empty vector group). Measurement data were represented as mean \pm standard deviation, unpaired $t-t e s t$ and one-way ANOVA with Tukey's post-hoc test. Cell experiments were repeated three times. 
EZH2 to the promoter region of CXCL3 and induced histone methylation, thus reducing the expression of CXCL3.

lncRNA MEG3 inhibits malignant behaviors and promotes cell apoptosis in gallbladder cancer cells by regulating CXCL3 expression. Following the aforementioned findings, we then managed to assess the effect of MEG3-mediated suppression of CXCL3 on malignant behaviors of gallbladder cancer cells. Our results of the CCK8 assay confirmed that CXCL3 overexpression could augment cell viability and reverse the inhibiting effect of MEG3 overexpression on cell malignant behaviors (Figure 5A). Flow cytometry detection then revealed that CXCL3 overexpression attenuated the apoptosis of the cells and negated the promoting effect of MEG3 overexpression on the apoptosis (Figure 5B). Consistently, the migration and invasion of the cells, as shown by Transwell results, were triggered by CXCL3 restoration, which could then be reversed when CXCL3 and MEG3 were simultaneously overexpressed (Figure 5C). Subsequently, we confirmed through western blot assay that CXCL3 overexpression led to the upregulated expression of pro-proliferative $\mathrm{Ki} 67$ and PCNA, anti-apoptotic $\mathrm{Bcl} 2$, and pro-invasive, pro-migratory E-cadherin, as well as downregulated expression of pro-apoptotic Bax and anti-invasive, anti-migratory MMP2 and MMP7, and simultaneous overexpression of MEG could reverse the aforementioned effects of CXCL3 overexpression alone (Figure 5D). Collectively, these results illuminated that IncRNA MEG3 repressed viability, migration, and invasion as well as promoted apoptosis of gallbladder cancer cells via downregulation of CXCL3 expression.

lncRNA MEG3 restricts tumor growth and liver metastasis by elevating CXCL3 expression in vivo. Next, we examined the tumorigenic ability of gallbladder cancer cells by subcutaneous tumorigenesis assay in nude mice. As shown in Figures $6 \mathrm{~A}-6 \mathrm{C}$, the volume and weight of tumor were restricted in response to lncRNA MEG3 overexpression alone, while after simultaneous treatment with lncRNA MEG3 overexpression and CXCL3 overexpression, the volume and weight of tumors were increased. The above results suggested that lncRNA MEG3 could repress tumor growth by inhibiting CXCL3 expression. Subsequently, we moved to investigate the relationship between tumor metastasis with lncRNA MEG3 and CXCL3 in nude mice. According to the results of $\mathrm{HE}$ staining, we illustrated that tumor metastasis was reduced after treatment with lncRNA MEG3 overexpression, while it was enhanced in the presence of lncRNA MEG3 overexpression and CXCL3 overexpression (Figure 6D). These results demonstrated that lncRNA MEG3 could control liver metastasis in gallbladder cancer by inhibiting CXCL3.

Subsequently, qRT-PCR results displayed that MEG3 upregulation, induced by pCDNA3.1-MEG3 treatment, led to reduced expression of CXCL3 and that additional oe-CXCL3 treatment restored the expression of CXCL3 (Figure 6E). Further, we performed immunohistochemistry to detect the expression level of CXCL3. The results showed that the positive expression rate of CXCL3 was decreased in response to lncRNA MEG3 overexpression, and simultaneous overexpression of lncRNA MEG3 and CXCL3 resulted in the increased positive expression of CXCL3 (Figure 6F), indicating that lncRNA MEG3 overexpression in tumor tissue could suppress CXCL3 expression.

\section{Discussion}

Currently, lncRNAs have been demonstrated to govern various biological processes during the development of many malignant tumors [6, 12, 13]. Intriguingly, lncRNA MEG3
A
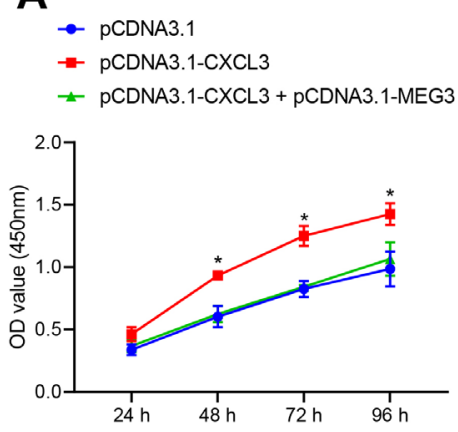

B
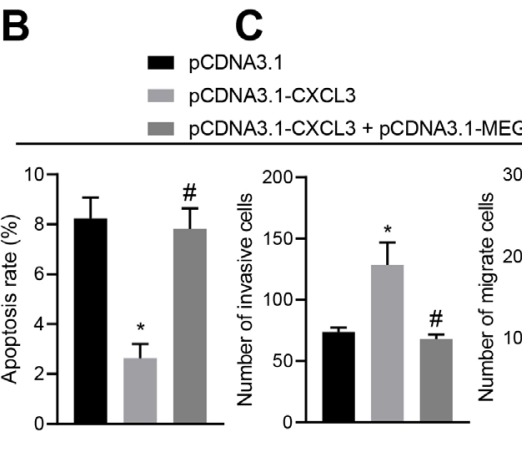

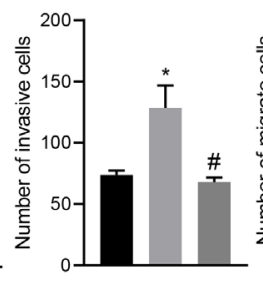

D \begin{tabular}{l} 
pCDNA3.1 \\
pCDNA3.1-CXCL3 \\
pCDNA3.1-CXCL3 + pCDNA3.1-MEG3 \\
\hline
\end{tabular}

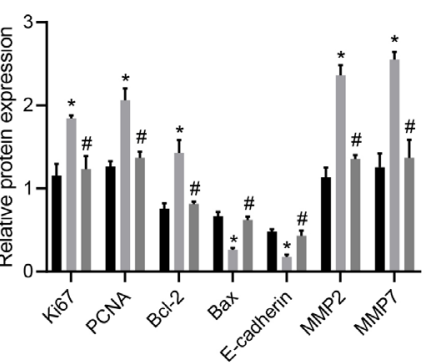

Figure 5. IncRNA MEG3 suppressed CXCL3 expression to impede malignant behaviors and stimulate apoptosis of gallbladder cancer cells. A) CCK-8 assay to detect cell viability in response to CXCL3 overexpression alone or in the combination with MEG3 overexpression. B) Flow cytometry to detect cell apoptosis in response to CXCL3 overexpression alone or in the combination with MEG3 overexpression. C) Transwell assay to detect cell invasion and migration in response to CXCL3 overexpression alone or in the combination with MEG3 overexpression. D) Western blot assay to detect the expression of proteins related to proliferation, migration, and invasion in gallbladder cancer cells in response to CXCL3 overexpression alone or in the combination with MEG3 overexpression. ${ }^{*} \mathrm{p}<0.05$ compared with the pCDNA3.1 group. Cell experiments were repeated three times. 


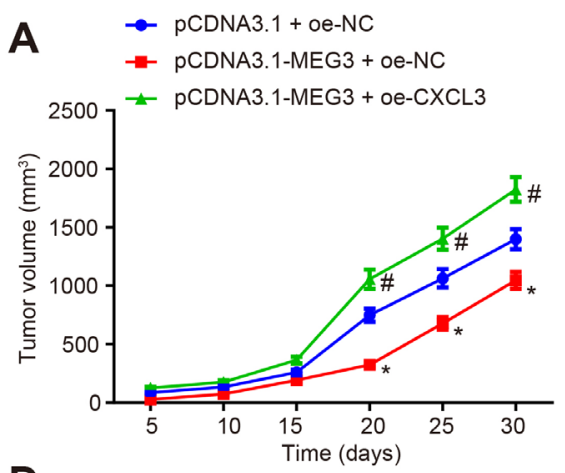

D
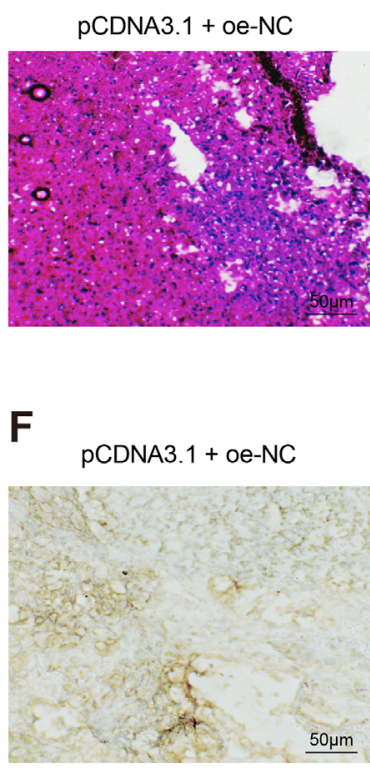

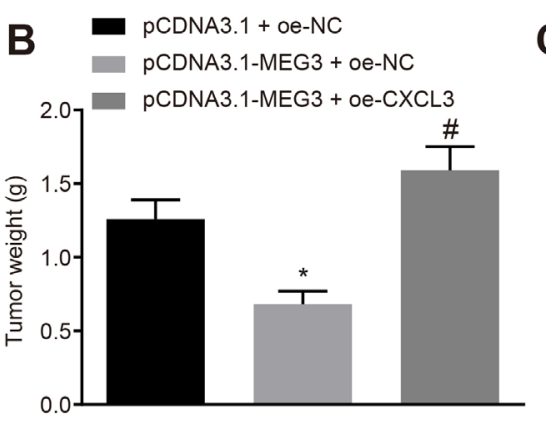

C

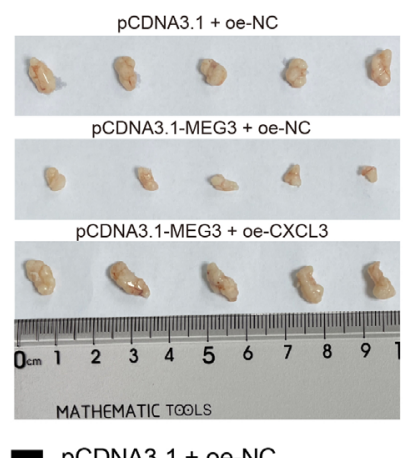

E
- pCDNA3.1 + oe-NC

- pCDNA3.1-MEG3 + oe-NC

pCDNA3.1-MEG3 + oe-CXCL3

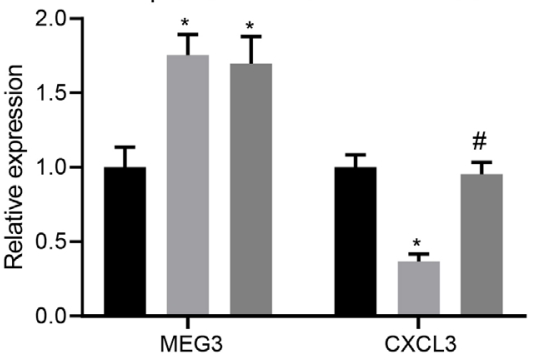

pCDNA3.1 + oe-NC

- pCDNA3.1-MEG3 + oe-NC

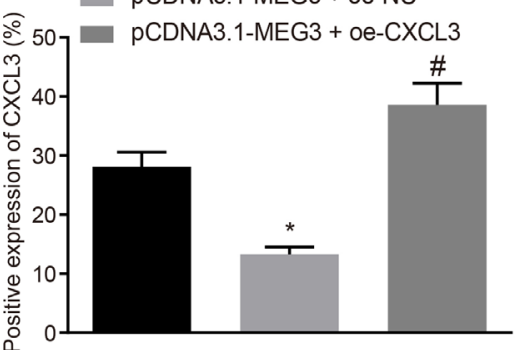

Figure 6. IncRNA MEG3 inhibited tumor growth and metastasis by suppressing the expression of CXCL3 in vivo. A) The quantitative analysis for tumor volume in response to lncRNA MEG3 overexpression alone or in the combination with CXCL3 overexpression. B) The quantitative analysis for tumor weight in response to lncRNA MEG3 overexpression alone or in the combination with CXCL3 overexpression. C) Representative images of tumors in response to lncRNA MEG3 overexpression alone or in the combination with CXCL3 overexpression. D) The HE staining results of liver tissue in response to lncRNA MEG3 overexpression alone or in the combination with CXCL3 overexpression. E) RT-qPCR measurement of the mRNA expression of MEG3 and CXCL3 in liver tissues in response to MEG3 overexpression alone or in the combination with CXCL3 overexpression. F) The expression of

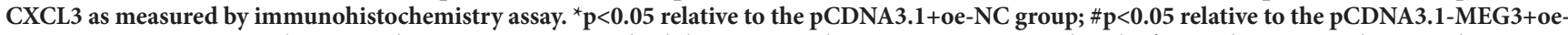
NC group. Measurement data were shown as mean \pm standard deviation, with one-way ANOVA with Tukey's post-hoc test, and repeated-measures ANOVA with Bonferroni post-hoc test. $n=6$

is expressed at a low level in gallbladder cancer tissues and it is closely related to patients' prognosis [18]. Nevertheless, specific downstream mechanisms of lncRNA MEG3 in gallbladder cancer growth and metastasis remain unestablished. Here, in the current study, we clarified that lncRNA MEG3 restricted the growth and metastasis of gallbladder cancer through EZH2-mediated CXCL3 histone methylation.

The human genome is mainly transcribed into lncRNAs, while only nearly $2 \%$ of genes are responsible for protein production $[11,26]$. Accumulating evidence has uncovered that lncRNAs frequently show dysregulated expression levels in various cancers, and confer pivotal roles in malignant cellular processes [27-29]. IncRNA MEG3, located at human chromosome $14 \mathrm{q} 32.3$, has been illustrated to serve as a tumor suppressor for many cancers [16, 30]. For example, lncRNA MEG3 overexpression could restrict the proliferation and cycle progression of tongue squamous cell carcinoma cells, and enhance apoptosis at the same time [31]. Besides, MEG3 is downregulated in gastric cancer patients and cell lines, and its expression level is related to gastric cancer progression [32]. Notably, lncRNA MEG3 has been reported to be associated with the development of gallbladder cancer [18], while 


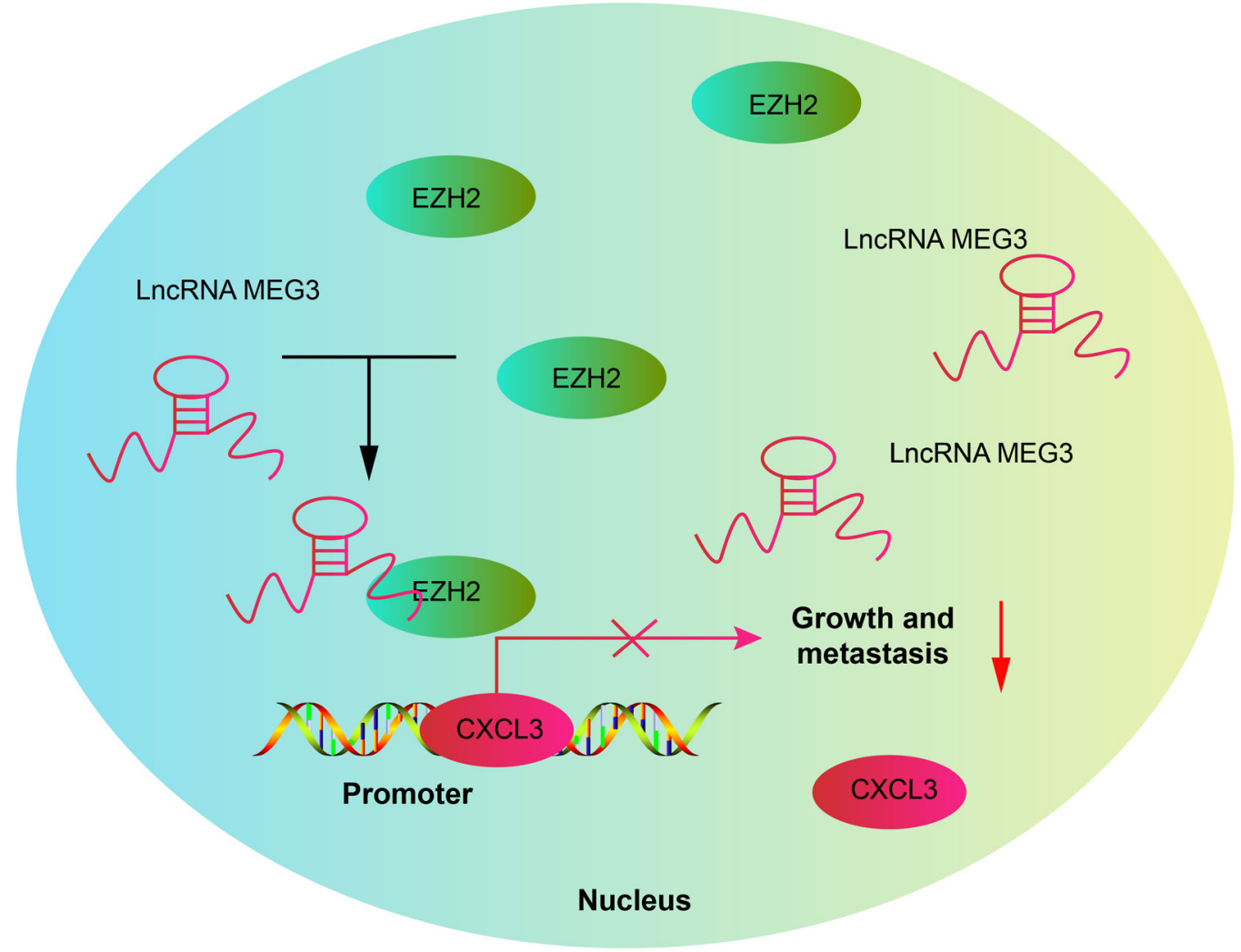

Figure 7. The mechanism graph of the regulatory network and function of lncRNA MEG3. IncRNA MEG3 binds to EZH2, and EZH2 catalyzes the H3K27 trimethylation of the CXCL3 promoter region. Further, lncRNA MEG3 suppresses tumor growth and metastasis by inhibiting the expression of CXCL3.

few studies have focused on its downstream mechanism. In this study, we revealed that lncRNA MEG3 is expressed at a low level in gallbladder cancer, and overexpression of lncRNA MEG3 could halt the development of gallbladder cancer.

In addition, it has been reported that IncRNA MEG3 could promote the degradation of EZH2 [18], so the effect of lncRNA MEG3 may be associated with EZH2. EZH2, a subunit member of PRC2, acts as a trigger of H3K27me3 trimethylation and further suppresses the expression of target genes, which is involved in tumorigenesis, senescence, cell proliferation, and apoptosis [19]. Interestingly, multiple lncRNAs modulate target genes by binding to EZH2 or impacting the stability of EZH2 [20,33]. Jin et al. suggested that $\ln$ cRNA MEG3 could affect EZH2 stability by enhancing its ubiquitination [18]. Likewise, in the current research, we indicated that lncRNA MEG3 could regulate gallbladder cancer progression by binding to EZH2 and further modulating the downstream genes.

Trimethylation of H3K27 is a critical epigenetic mechanism catalyzed by EZH2 [34]. Wang et al. has demonstrated that IncRNA MEG3 enhances the expression of $\operatorname{Rad} 21$, Smc3, and $\operatorname{Sin} 3 \alpha$ via EZH2-induced H3K27 trimethylation [35]. H3K27me3 trimethylation is also related to the progression of malignant tumors. For example, lncRNA
MEG3 restricts the development of prostate cancer by promoting $\mathrm{H} 3 \mathrm{~K} 27$ trimethylation of EN2 via interaction with EZH2 [34]. IncRNA MEG3 suppresses proliferation and invasion of gallbladder cancer through EZH2 degradation and $\mathrm{H} 3 \mathrm{~K} 27 \mathrm{me} 3$ trimethylation [18]. In our study, we found that MEG3 downregulated CXCL3 expression by triggering EZH2-mediated histone methylation in the CXCL3 promoter region, and we further demonstrated that MEG3 inhibited malignant behaviors and promoted cell apoptosis in gallbladder cancer cells by reducing CXCL3 expression. lncRNA MEG3 inhibited the progression of gallbladder cancer by promoting EZH2-mediated H3K27 trimethylation of CXCL3. Moreover, CXCL3 is overexpressed in various cancers, which is a potential suppressor for multiple malignancies $[22,24]$. CXCL3 is suggested to be a promising target for various cancer, such as breast cancer [24, 36], uterine cervical cancer [22], and prostate cancer [37].

To sum up, our data in this study elucidated that lncRNA MEG3 could bind to EZH2 and further inhibit CXCL3 expression by mediating the $\mathrm{H} 3 \mathrm{~K} 27$ trimethylation of CXCL3; overexpression of lncRNA MEG3 could attenuate cancer cell malignant behaviors in vitro and halt tumor growth and metastasis in vivo in gallbladder cancer through suppressing CXCL3 expression (Figure 7). Based on results 
acquired in the present study, we demonstrated that lncRNA MEG3 could restrict gallbladder cancer progression by modulating EZH2-induced CXCL3 methylation, which may provide a theoretical basis and possible therapeutic targets for the diagnosis and treatment of gallbladder cancer.

Supplementary information is available in the online version of the paper.

Acknowledgments: This work was supported by The Plan to the Introduction of Clinical Medical Expert Teams of Xuzhou City (No. 2018TD011).

\section{References}

[1] TORRE LA, BRAY F, SIEGEL RL, FERLAY J, LORTETTIEULENT J et al. Global cancer statistics, 2012. CA Cancer J Clin 2015; 65: 87-108. http://doi.org/10.3322/caac.21262

[2] ZHU AX, HONG TS, HEZEL AF, KOOBY DA. Current management of gallbladder carcinoma. Oncologist 2010; 15: 168-181. http://doi.org/10.1634/theoncologist.2009-0302

[3] HU YP, JIN YP, WU XS, YANG Y, LI YS et al. LncRNA-HG$\mathrm{BC}$ stabilized by HuR promotes gallbladder cancer progression by regulating miR-502-3p/SET/AKT axis. Mol Cancer 2019; 18: 167. http://doi.org/10.1186/s12943-019-1097-9

[4] WU XS, SHI LB, LI ML, DING Q, WENG H et al. Evaluation of two inflammation-based prognostic scores in patients with resectable gallbladder carcinoma. Ann Surg Oncol 2014; 21: 449-457. http://doi.org/10.1245/s10434-013-3292-z

[5] LI M, ZHANG Z, LI X, YE J, WU X et al. Whole-exome and targeted gene sequencing of gallbladder carcinoma identifies recurrent mutations in the ErbB pathway. Nat Genet 2014; 46: 872-876. http://doi.org/10.1038/ng.3030

[6] SICKLICK JK, FANTA PT, SHIMABUKURO K, KURZROCK R. Genomics of gallbladder cancer: the case for biomarker-driven clinical trial design. Cancer Metastasis Rev 2016; 35: 263-275. http://doi.org/10.1007/s10555-016-9602-8

[7] HAN M, WANG S, FRITAH S, WANG X, ZHOU W et al. Interfering with long non-coding RNA MIR22HG processing inhibits glioblastoma progression through suppression of Wnt/beta-catenin signalling. Brain 2020; 143: 512-530. http://doi.org/10.1093/brain/awz406

[8] LI DY, BUSCH A, JIN H, CHERNOGUBOVA E, PELISEK $\mathrm{J}$ et al. H19 Induces Abdominal Aortic Aneurysm Development and Progression. Circulation 2018; 138: 1551-1568. http://doi.org/10.1161/CIRCULATIONAHA.117.032184

[9] WANG M, MAO C, OUYANG L, LIU Y, LAI W et al. Long noncoding RNA LINC00336 inhibits ferroptosis in lung cancer by functioning as a competing endogenous RNA. Cell Death Differ 2019; 26: 2329-2343. http://doi.org/10.1038/ s41418-019-0304-y

[10] YANG R, LIU N, CHEN L, JIANG Y, SHI Y et al. GIAT4RA functions as a tumor suppressor in non-small cell lung cancer by counteracting Uchl3-mediated deubiquitination of LSH. Oncogene 2019; 38: 7133-7145. http://doi.org/10.1038/ s41388-019-0909-0
[11] SCHMITT AM, CHANG HY. Long Noncoding RNAs in Cancer Pathways. Cancer Cell 2016; 29: 452-463. http://doi. org/10.1016/j.ccell.2016.03.010

[12] XUE X, YANG YA, ZHANG A, FONG KW, KIM J et al. LncRNA HOTAIR enhances ER signaling and confers tamoxifen resistance in breast cancer. Oncogene 2016; 35: 27462755. http://doi.org/10.1038/onc.2015.340

[13] PRENSNER JR, CHEN W, IYER MK, CAO Q, MA T et al. PCAT-1, a long noncoding RNA, regulates BRCA2 and controls homologous recombination in cancer. Cancer Res 2014; 74: 1651-1660. http://doi.org/10.1158/0008-5472.CAN-133159

[14] BARNHILL LM, WILLIAMS RT, COHEN O, KIM Y, BATOVA A et al. High expression of CAI2, a 9p21-embedded long noncoding RNA, contributes to advanced-stage neuroblastoma. Cancer Res 2014; 74: 3753-3763. http://doi. org/10.1158/0008-5472.CAN-13-3447

[15] MAO C, WANG X, LIU Y, WANG M, YAN B et al. A G3BP1Interacting lncRNA Promotes Ferroptosis and Apoptosis in Cancer via Nuclear Sequestration of p53. Cancer Res 2018; 78: 3484-3496. http://doi.org/10.1158/0008-5472.CAN-173454

[16] ZHOU C, HUANG C, WANG J, HUANG H, LI J et al. LncRNA MEG3 downregulation mediated by DNMT3b contributes to nickel malignant transformation of human bronchial epithelial cells via modulating PHLPP1 transcription and HIF-1alpha translation. Oncogene 2017; 36: 3878-3889. http://doi.org/10.1038/onc.2017.14

[17] TERASHIMA M, TANGE S, ISHIMURA A, SUZUKI T. MEG3 Long Noncoding RNA Contributes to the Epigenetic Regulation of Epithelial-Mesenchymal Transition in Lung Cancer Cell Lines. J Biol Chem 2017; 292: 82-99. http://doi. org/10.1074/jbc.M116.750950

[18] JIN L, CAI Q, WANG S, WANG S, MONDAL T et al. Long noncoding RNA MEG3 regulates LATS2 by promoting the ubiquitination of EZH2 and inhibits proliferation and invasion in gallbladder cancer. Cell Death Dis 2018; 9: 1017. http://doi.org/10.1038/s41419-018-1064-1

[19] SAUVAGEAU M, SAUVAGEAU G. Polycomb group proteins: multi-faceted regulators of somatic stem cells and cancer. Cell Stem Cell 2010; 7: 299-313. http://doi.org/10.1016/j. stem.2010.08.002

[20] CHEN Q, CAI J, WANG Q, WANG Y, LIU M et al. Long Noncoding RNA NEAT1, Regulated by the EGFR Pathway, Contributes to Glioblastoma Progression Through the WNT/beta-Catenin Pathway by Scaffolding EZH2. Clin Cancer Res 2018; 24: 684-695. http://doi.org/10.1158/10780432.CCR-17-0605

[21] SANLI I, LALEVEE S, CAMMISA M, PERRIN A, RAGE $F$ et al. Meg3 Non-coding RNA Expression Controls Imprinting by Preventing Transcriptional Upregulation in cis. Cell Rep 2018; 23: 337-348. http://doi.org/10.1016/j.celrep.2018.03.044

[22] QI YL, LI Y, MAN XX, SUI HY, ZHAO XL et al. CXCL3 overexpression promotes the tumorigenic potential of uterine cervical cancer cells via the MAPK/ERK pathway. J Cell Physiol 2020; 235: 4756-4765. http://doi.org/10.1002/ jcp. 29353 
[23] CECCARELLI M, MICHELI L, TIRONE F. Suppression of Medulloblastoma Lesions by Forced Migration of Preneoplastic Precursor Cells with Intracerebellar Administration of the Chemokine Cxcl3. Front Pharmacol 2016; 7: 484. http://doi.org/10.3389/fphar.2016.00484

[24] SEE AL, CHONG PK, LU SY, LIM YP. CXCL3 is a potential target for breast cancer metastasis. Curr Cancer Drug Targets 2014; 14: 294-309. http://doi.org/10.2174/15680096146 66140305222328

[25] YADAV S, TELLA SH, KOMMALAPATI A, MARA K, PRASAI K et al. A Novel Clinically Based Staging System for Gallbladder Cancer. J Natl Compr Canc Netw 2020; 18 : 151-159. http://doi.org/10.6004/jnccn.2019.7357

[26] LOURO R, SMIRNOVA AS, VERJOVSKI-ALMEIDA S. Long intronic noncoding RNA transcription: expression noise or expression choice? Genomics 2009; 93: 291-298. http://doi.org/10.1016/j.ygeno.2008.11.009

[27] WAPINSKI O, CHANG HY. Long noncoding RNAs and human disease. Trends Cell Biol 2011; 21: 354-361. http://doi. org/10.1016/j.tcb.2011.04.001

[28] BUNCH H. Gene regulation of mammalian long non-coding RNA. Mol Genet Genomics 2018; 293: 1-15. http://doi. org/10.1007/s00438-017-1370-9

[29] FATIMA F, NAWAZ M. Vesiculated Long Non-Coding RNAs: Offshore Packages Deciphering Trans-Regulation between Cells, Cancer Progression and Resistance to Therapies. Noncoding RNA 2017; 3: http://doi.org/10.3390/ ncrna3010010

[30] ZHANG CY, YU MS, LI X, ZHANG Z, HAN CR et al. Overexpression of long non-coding RNA MEG3 suppresses breast cancer cell proliferation, invasion, and angiogenesis through AKT pathway. Tumour Biol 2017; 39: 1010428317701311. http://doi.org/10.1177/1010428317701311
[31] JIA LF, WEI SB, GAN YH, GUO Y, GONG K et al. Expression, regulation and roles of miR-26a and MEG3 in tongue squamous cell carcinoma. Int J Cancer 2014; 135: 2282-2293. http://doi.org/10.1002/ijc.28667

[32] PENG W, SI S, ZHANG Q, LI C, ZHAO F et al. Long noncoding RNA MEG3 functions as a competing endogenous RNA to regulate gastric cancer progression. J Exp Clin Cancer Res 2015; 34: 79. http://doi.org/10.1186/s13046-0150197-7

[33] LI Z, HOU P, FAN D, DONG M, MA M et al. The degradation of EZH2 mediated by lncRNA ANCR attenuated the invasion and metastasis of breast cancer. Cell Death Differ 2017; 24: 59-71. http://doi.org/10.1038/cdd.2016.95

[34] ZHOU Y, YANG H, XIA W, CUI L, XU R et al. LncRNA MEG3 inhibits the progression of prostate cancer by facilitating H3K27 trimethylation of EN2 through binding to EZH2. J Biochem 2020; 167: 295-301. http://doi.org/10.1093/jb/ mvz097

[35] WANG N, ZHU Y, XIE M, WANG L, JIN F et al. Long Noncoding RNA Meg3 Regulates Mafa Expression in Mouse Beta Cells by Inactivating Rad21, Smc3 or Sin3alpha. Cell Physiol Biochem 2018; 45: 2031-2043. http://doi. org/10.1159/000487983

[36] BIECHE I, CHAVEY C, ANDRIEU C, BUSSON M, VACHER $S$ et al. CXC chemokines located in the 4q21 region are up-regulated in breast cancer. Endocr Relat Cancer 2007; 14: 1039-1052. http://doi.org/10.1677/erc.1.01301

[37] KOGAN-SAKIN I, COHEN M, PALAND N, MADAR S, SOLOMON H et al. Prostate stromal cells produce CXCL1, CXCL-2, CXCL-3 and IL-8 in response to epithelia-secreted IL-1. Carcinogenesis 2009; 30: 698-705. http://doi. org/10.1093/carcin/bgp043 
https://doi.org/10.4149/neo_2022_210726N1046

\section{Tumor suppressive lncRNA MEG3 binds to EZH2 and enhances CXCL3 methylation in gallbladder cancer}

De-Qiang LI*, Yi-Ren DING*, Jin-Hui CHE, Zhan SU, Wei-Zhong YANG, Lu XU, Yun-Jiu LI, Hai-Hong WANG*, Wu-Yuan ZHOU*

\section{Supplementary Information}

Supplementary Table S1. Primer sequences for the qRT-PCR assay.

\begin{tabular}{ll}
\hline Gene & Primer sequence \\
\hline MEG3 & Forward: 5'-GAGTGTTTCCCTCCCCAAGG-3' \\
& Reverse: 5'-GCGTGCCTTTGGTGATTCAG-3' \\
CXCL3 & Forward: 5'-ACCGAAGTCATAGCCACACTCAAG-3' \\
& Reverse: 5'-ACTTCTCTCCTGTCAGTTGGTGCT-3' \\
\multirow{3}{*}{-actin } & Forward: 5'-ACACTGTGTGCCCATCTACGAGG-3' \\
& Reverse: 5'-AGGGGCCGGACTCGTCGTCATACT-3' \\
\hline
\end{tabular}

Supplementary Table S2. Primer sequences for the ChIP assay.

\begin{tabular}{ll}
\hline Gene & Primer sequence \\
\hline CXCL3 & Forward: 5'-GGTCCCCAGCAAATCTGATA-3' \\
& Reverse: 5'-GGTCCCCAGCAAATCTGATA-3' \\
\hline
\end{tabular}

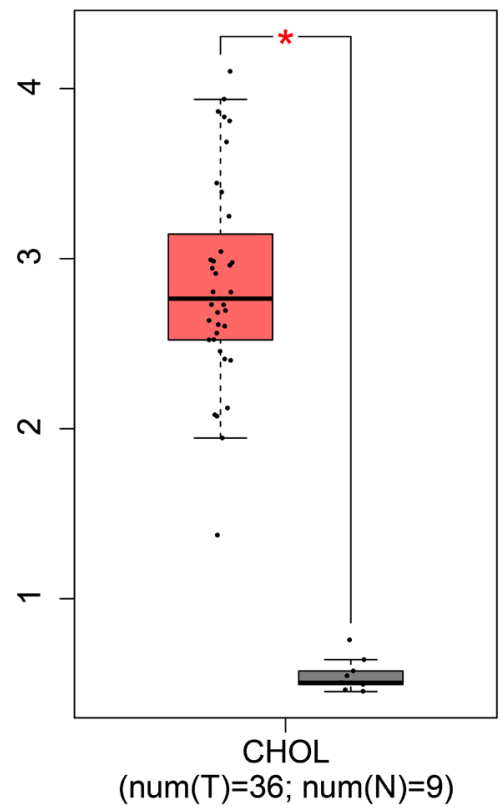

Supplementary Figure S1. GEPIA database-based analysis of the expression of EZH2 in gallbladder cancer samples. A box plot for the expression of EZH2 in gallbladder cancer samples and normal samples $\left({ }^{*}|\log 2 \mathrm{FC}|>1, \mathrm{p}<0.01\right)$. 